\title{
Dynamical behaviour of epidemiological models with sub-optimal immunity and nonlinear incidence
}

Received: 3 December 2004 / Revisied version: 9 March 2005

Published online: $\mathbf{\square} 2005$ - (c) Springer-Verlag 2005

Abstract. In this paper we analyze the dynamics of two families of epidemiological models which correspond to transitions from the $S I R$ (susceptible-infectious-resistant) to the $S I S$ (susceptible-infectious-susceptible) frameworks. In these models we assume that the force of infection is a nonlinear function of density of infectious individuals, $I$. Conditions for the existence of backwards bifurcations, oscillations and Bogdanov-Takens points are given.

\section{Introduction}

The simplest mathematical models for infectious disease dynamics are based on averages. Hosts are classified as susceptible $(S)$, infectious $(I)$, or recovered $(R)$ with immunity $[13,4]$. The $S I R$ and $S I S$ frameworks are used to model two extreme situations: in $S I R$, immunity is assumed to be fully protective and prevents any reinfection; in SIS, immunity is assumed not to protect against reinfection and previous infection does not alter the risk of subsequent reinfection. Most real infections lie somewhere between these two extremes, and different model families can be constructed to represent specific biological mechanisms of sub-optimal immunity $([9,25])$.

For a transmissible pathogen the risk of infection increases with the density of infectious individuals in the population, i.e. the risk of a susceptible individual becoming infected increases with the proportion of the population that are infected. A linear relationship is the most common assumption so that doubling the number of infectious individuals doubles the rate of infection to each susceptible. However, it has long been established in the mathematical biology literature that model behaviour is sensitive to deviations from this assumption (see [14] and references therein).

In diseases that do not elicit significant protective immunity (such as chronic infections or in the SIS model), nonlinear incidence functions tend to generate bistable behaviour as a positive feedback maintains high or low endemicities depending on the initial infection density $([17,5,24])$. The same mechanism is also capable

M.G.M. Gomes: Instituto Gulbenkian de Ciência, Apartado 14, 2781-901 Oeiras, Portugal

A. Margheri, C. Rebelo: Fac. Ciências de Lisboa and Centro de Matemática e Aplicações Fundamentais , Av. Prof. Gama Pinto 2,1649-003 Lisboa, Portugal, Supported by FCT.

G.F. Medley: Ecology and Epidemiology Group, Department of Biological Sciences, University of Warwick, Coventry CV4 7AL, UK

\begin{tabular}{|l|l|l|l|l|l|l}
2 & $\mathbf{2}|\mathbf{5}| \mathbf{0}|\mathbf{3}| \mathbf{3}|\mathbf{1}| \mathbf{B}$ & $\begin{array}{l}\text { Dispatch: 29/4/2005 } \\
\text { Total pages: 17 } \\
\text { Disk Recived } \square \\
\text { Disk Used } \square\end{array}$ & $\begin{array}{l}\text { Journal: J. Math. Biol. } \\
\text { Not Used } \square \\
\text { Corrupted } \square \\
\text { Mismatch } \square\end{array}$ \\
\hline Jour. No $\mid$ Ms. No. &
\end{tabular}


of sustaining a periodic epidemic behaviour $([1,22])$ if there is significant time delay in the replenishment of the susceptibility pool (such as in the SIR model where susceptibles are supplied by births). Intermediate scenarios (such as the SI RS model) can accommodate either behaviour depending on the formulation and parameter values $([14,12,11])$. In this paper we analyse the epidemiological consequences of nonlinear incidence rates in models that bridge the SIR and SIS extremes. Two distinct mechanisms (temporary immunity and partial immunity) are implemented and systematically analysed. In both cases Bogdanov-Takens points and associated homoclinic bifurcations are detected, implying a tendency for intermittent epidemic behaviour. Despite increasing awareness of these nonlinear effects, models aimed at describing the dynamics of specific diseases predominantly continue to implement the force of infection as increasing linearly with the prevalence of infectious individuals. This is likely to be attributed to difficulties in deriving nonlinear functional forms for the force of infection from surveillance data [15] or from mechanistic assumptions.

Transmission of infection requires that susceptible individuals encounter the infectious agent, and this is typically through contact with infectious individuals. There is good documentation on dose-response relationships from experimental studies in terms of infection, disease, and immunity. The majority of such empirical studies conclude that there is a minimal dose required to induce a response, and above this threshold the probability of response increases nonlinearly with the dose of exposure $[8,18]$. To incorporate this observation into a model of transmission we need a scheme that processes the shedding and spread of infectious agent to determine the average dose challenging a susceptible. This is a rather complex mechanism, and it depends on measures of infectious agent shed by infectious individuals and on contact patterns of the host population (modulated by the mode of transmission).

A first obstacle is the scarcity of information on the amount of infectious agent shed by infectious individuals. Is there a minimal dose that can be shed, and what controls this quantity? A second obstacle is to absorb the effects of realistic contact patterns into simple models. What is the role of duration, intensity and multitude of exposures? How should transmission be modelled, and in what unit? We refer two extreme approaches: (1) the most standard approach considers the infectious host as the unit of transmission, and transmission as a mass action process, $\beta I S$, given that $\beta=p c$ where $c$ is the contact rate between individuals and $p$ is the probability that a contact between a susceptible and infectious results in a new infection; (2) an alternative approach is to work with units of infectious agent [21] and model transmission as $\beta(v) S$, where $v$ is a measure of infectious agent and $\beta(v)$ is a sigmoidal function to represent dose-response relationships observed in infection experiments. A problem with the first approach is to ignore dose-response relationships. A problem with the second approach is to consider that a dose of infectious agent is diluted into the environment the moment it is shed by an individual, and this may induce artificial effects when the prevalence of infection is low. More elaborate individual-based models are perhaps the most accurate frameworks to encapsulate infectious agent units into a model whose transmission unit is the infectious host [23]. But there is a high cost in terms of simplicity and mathematical tractability. 
Collapsing the richness of these models into a functional form for a transmission rate that can be incorporated in the $S I R$ model (and its extensions) is certainly worth pursuing. Encouragement continues to arise from empirical relationships between intensity of exposure, infection and disease outcomes, and efficiency of spread $[2$, 20]. In the mean time, the behaviour of the generic $S I R$ model (where the functional form for the transmission rate can be fairly general) is worth characterizing.

\section{Sub-optimal immunity and nonlinear incidence}

Following [9] we consider two families of models that correspond to a transition between $S I R$ and $S I S$ frameworks. These are parametrized by $\sigma$, such that $\sigma=0$ corresponds to the $S I R$ model and $\sigma=1$ corresponds to the $S I S$ model. In one of the families immunity wanes over time (temporary immunity - TI), while in the other immunity is not fully protective but reduces the risk of subsequent infections by a certain factor (partial immunity - $P I$ ). The models are described by the following systems of equations and represented diagrammatically in Figure 1.

$$
(T I)\left\{\begin{array}{l}
\frac{\partial S}{\partial T}=\mu-\lambda(I) S+\sigma \tau(I+R)-\mu S \\
\frac{\partial I}{\partial T}=\lambda(I) S-(\tau+\mu) I \\
\frac{\partial R}{\partial T}=(1-\sigma) \tau I-(\sigma \tau+\mu) R
\end{array}\right.
$$

(a)

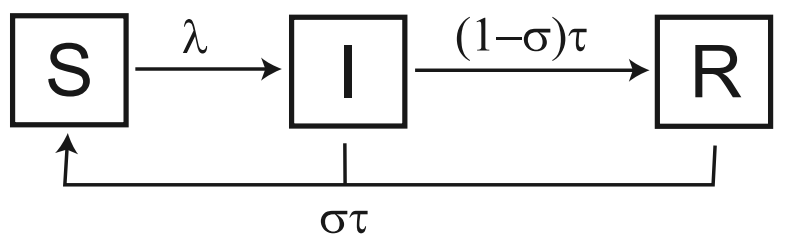

(b)

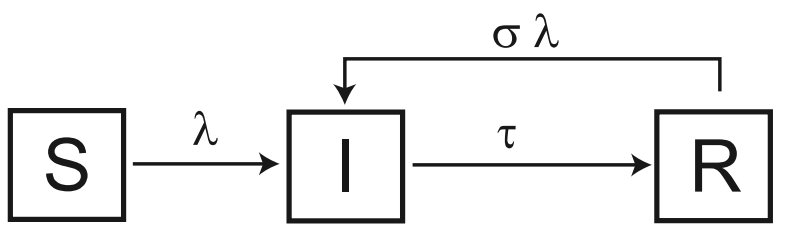

Fig. 1. Diagrammatic representation of the temporary immunity $(T I)$ and partial immunity $(P I)$ models, parametrized by $0 \leq \sigma \leq 1$. The two models coincide at the extremes $\sigma=0$ ( $S I R$ model) and $\sigma=1$ (SIS model). Note that $(T I)$ is not the SIRS model, and the latter would not fulfill our requirement of convergence to $S I S$ as the duration of immunity becomes shorter 
and

$$
(P I)\left\{\begin{array}{l}
\frac{\partial S}{\partial T}=\mu-\lambda(I) S-\mu S \\
\frac{\partial I}{\partial T}=\lambda(I)(S+\sigma R)-(\tau+\mu) I \\
\frac{\partial R}{\partial T}=\tau I-\sigma \lambda(I) R-\mu R
\end{array}\right.
$$

where $0 \leq \sigma \leq 1$. In the systems above, $\mu$ is the death rate (and, equally, the birth rate), $\tau$ is the rate of recovery from infection and $\lambda(I)$ is the force of infection. The last equation can be omitted in both models as $S+I+R=1$.

The models can be non-dimensionalized by representing time in units of the average duration of infection, $D$, that is by considering $t=\frac{T}{D}$ where $D=\frac{1}{\tau+\mu}$. In this way we obtain

$$
\text { (TI) }\left\{\begin{array}{l}
\dot{S}=\alpha-(\Lambda+\alpha) S \\
\dot{I}=\Lambda S-I
\end{array}\right.
$$

with $\alpha=\alpha(\sigma)=e+(1-e) \sigma$ and

$$
(P I)\left\{\begin{array}{l}
\dot{S}=e-\Lambda S-e S \\
\dot{I}=\Lambda(S+\sigma(1-S-I))-I
\end{array}\right.
$$

where the dot means the derivative with respect to time, $t$, and $\Lambda(I)=\lambda(I) D$. The parameter $e$ represents the rate of birth and death (here considered equal) in units of average duration of infection. Throughout this paper, illustrative numerical results are obtained assuming $e=0.0012$, which can represent an infection of one month duration in a host population with 70 years life span. Shorter infections or longer host life span would lead to smaller $e$, while the opposite would lead to larger $e$. In the following we will always consider the rescaled models.

In both systems the set $\left\{(S, I) \in \mathbb{R}_{0}^{+} \times \mathbb{R}_{0}^{+}: S+I \leq 1\right\}\left(\mathbb{R}_{0}^{+}=[0,+\infty[)\right.$ is positively invariant and hence in the following we study the systems $(T I)$ and $(P I)$ restricted to that set. The force of infection (or per capita rate of infection) $\Lambda$ is a function of the proportion of infectious individuals, $I$, parametrized by the basic reproduction number, $R_{0}$. The linear case, $\Lambda=R_{0} I$, has been thoroughly analysed in [9] and used to explore the impact of vaccination programmes in both scenarios. Here we consider a nonlinear force of infection of the general form

$$
\Lambda:=r(I) I:=\left(R_{0}+h(I)\right) I,
$$

where $r$ is a transmission coefficient affected by the density of infected hosts. The function $h: \mathbb{R}_{0}^{+} \rightarrow \mathbb{R}$ is $\mathcal{C}^{1}$ and increasing such that $h(0)=0$. Note that assuming $h$ increasing corresponds to considering that the risk of infection increases with the 
intensity of exposure and that when $h \equiv 0$ we have the standard force of infection, $\Lambda=R_{0} I$.

In this section we state and demonstrate results that are common to both $(T I)$ and $(P I)$ systems, and the next two sections are concerned with further analysis of each system separately. Although the results are proved in a general context, illustrations will be provided using a specific functional form for $h$ as follows

$$
h(I):=R_{1} \frac{k I}{1+k I},
$$

where $R_{1}$ is a parameter that sets the amplitude of variation of the transmission coefficient $r$ (between $R_{0}$ and $R_{0}+R_{1}$ ), and $k$ controls the steepness of $r$ at the origin (note that $r^{\prime}(0)=h^{\prime}(0)=R_{1} k$ ). As we will see this parameter is crucial to the sustainability of oscillations. The function $k I /(1+k I)$ is shown in Figure 2 for different values of $k$.

For simplicity, and as no confusion arises, we omit the dependence of $\Lambda, r$ and $h$ on $I$ and $R_{0}$, and write simply $\Lambda=r I=\left(R_{0}+h\right) I$. In order to describe the sets of nontrivial equilibria, we introduce the auxiliary functions

$$
\begin{aligned}
\eta_{T}(I) & =\frac{\alpha(\sigma)}{\alpha(\sigma)-I} \text { and } \eta_{P}(I) \\
& =\frac{2 e}{\sqrt{[I(1+e \sigma)-e]^{2}+4 \sigma e\left(I-I^{2}\right)}+e-I(1+e \sigma)} .
\end{aligned}
$$

We have the following

Theorem 1. For each $0 \leq \sigma \leq 1$ the disease-free steady state $(S=1$ and $I=0)$ is an equilibrium (trivial equilibrium) for both systems (TI) and (PI). This equilibrium is locally asymptotically stable for $R_{0}<1$ and unstable for $R_{0}>1$.

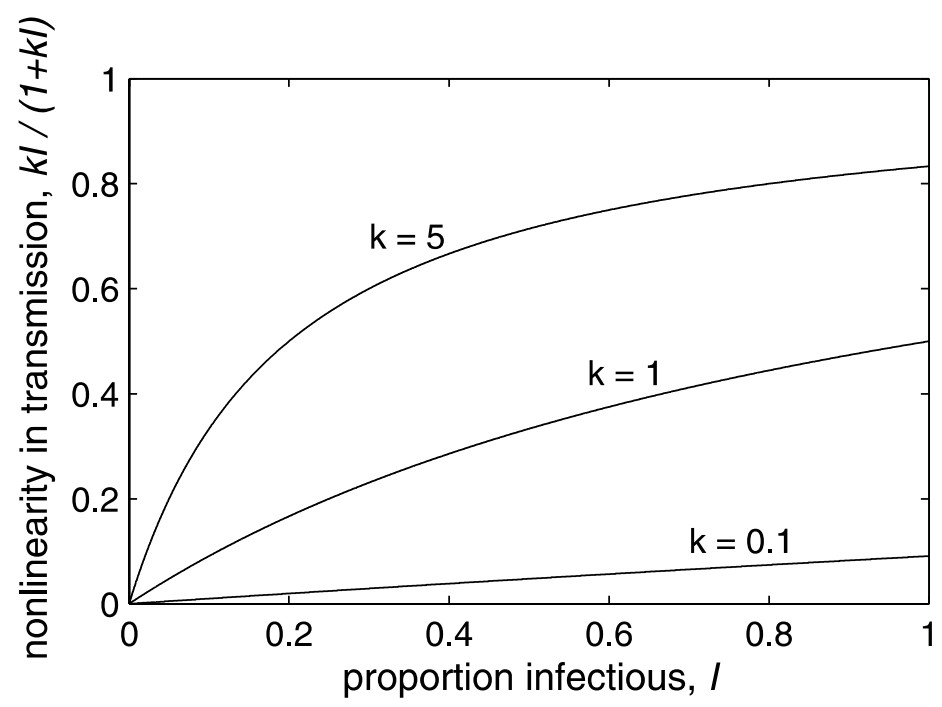

Fig. 2. Nonlinearity in the transmission coefficient, $k I /(1+k I)$, for $k=0.1,1,5$ 
At $R_{0}=1$ a branch of nontrivial equilibria emanates from the trivial one. The nontrivial equilibria are the pairs $(S, I)$ such that

$$
\begin{aligned}
& S=\frac{\alpha(\sigma)}{\Lambda+\alpha(\sigma)} \text { and } r(I)=\eta_{T}(I) \text { in the case of system }(T I), \\
& S=\frac{e}{\Lambda+e} \text { and } r(I)=\eta_{P}(I) \text { in the case of system }(P I) .
\end{aligned}
$$

In both systems, for each $R_{0}>1$ there is at least one nontrivial equilibrium.

The bifurcation is backwards if

$$
h^{\prime}(0)>\frac{1}{\alpha(\sigma)} \text { in the case of system }(T I),
$$

and if

$$
h^{\prime}(0)>\frac{1-\sigma}{e}+\sigma \text { in the case of system }(P I) .
$$

Proof. The eigenvalues of the Jacobian matrix evaluated at the trivial equilibrium are $-\alpha$ and $R_{0}-1$ in the case of the $(T I)$ model and $-e$ and $R_{0}-1$ in the case of the $(P I)$ model. Hence the first part of the result immediately follows.

The formulas which give implicitly the equilibria are easily obtained from the differential equations. Note that as $h(I)>0$ for each $I>0$, if $(S, I)$ is an equilibrium then $I \in\left[0, I^{\star}\left[\right.\right.$, where, in the case of system $(T I), I^{\star}=\alpha$ and, in the case of system $(P I), I^{\star}=e$ if $\sigma=0$ and $I^{\star}=1$ when $0<\sigma \leq 1$.

The existence of at least a nontrivial equilibrium when $R_{0}>1$ follows immediately from the fact that when $R_{0}>1$ the functions $R_{0}+h(I)-\eta_{P}(I)$ and $R_{0}+h(I)-\eta_{T}(I)$ change sign when $I \in\left[0, I^{\star}[\right.$.

Note that in the branch of nontrivial equilibria $R_{0}$ can be written as a function $R_{0}(I)$. Also when $I \rightarrow I^{\star}, R_{0}(I) \rightarrow+\infty$ and $R_{0}^{\prime}(I) \rightarrow+\infty$. Hence for $R_{0}$ sufficiently large the branch can be written as a graph of a function $I\left(R_{0}\right)$ and thus the nontrivial equilibrium is unique.

Moreover $R_{0}^{\prime}(0)=\frac{1-\alpha h^{\prime}(0)}{\alpha}$ in the case of system $(T I)$ and $R_{0}^{\prime}(0)=$ $\frac{1-\sigma}{e}+\sigma-h^{\prime}(0)$ in the case of system $(P I)$. Hence the theorem follows.

Corollary 1. For each $0 \leq \sigma \leq 1$ the disease-free equilibrium $(S=1$ and $I=0)$ is globally asymptotically stable whenever it is the unique equilibrium of the system.

Proof. Under the conditions of the Corollary, $R_{0}<1$ and the trivial equilibrium is locally asymptotically stable. Now the result follows from the fact that in this case as a consequence of the Poincaré-Bendixson theorem the trivial equilibrium is also an attractor of all orbits. 


\section{The temporary immunity model}

In this section we will focus on system (TI). Figure 3 shows the branches of equilibria, as a function of $R_{0}$. The three plots used a function $h$ as in (2) with $R_{1}=2$ and three values of $k$, namely $k=0,2,5$. Within each plot five curves are represented corresponding to different values of $\sigma$. Here we can see an evidence for backward bifurcations as the parameter $k$ increases. We can also note that backward bifurcation are more evident towards the SIS limit of this family of models. The branch of nontrivial equilibria is more generally characterised as follows.

Theorem 2. Assume $h^{\prime \prime}(I)<0, \forall I$. Then for each $\sigma$ and $R_{0}$ there are at most two nontrivial equilibria of system $(T I)$. Hence

i) if(5) is satisfied there exists $R_{1}<1$ such that for each $R_{0}$ satisfying $R_{1}<R_{0}<$ 1 there are two nontrivial equilibria for system $(T I)$ and if $R_{0}=R_{1}$ or $R_{0} \geq 1$ there is exactly one,

ii) otherwise for each $R_{0} \leq 1$ there are no nontrivial equilibria for system (TI) and for each $R_{0}>1$ there is exactly one.

Proof. The result follows from the fact that as we assume $r^{\prime \prime}<0$, we have that for each fixed $R_{0}$ the function $r(I)-\eta_{T}(I)$ is strictly concave and hence it can assume at most two zeros.

The existence of solutions which oscillate is one important characteristic of the systems with nonlinear incidence. In the next result we prove that assuming $h^{\prime \prime}(I)<0$ and $h^{\prime \prime \prime}(I)>0$ there is at least a limit cycle surrounding the nontrivial equilibrium provided that $h^{\prime}(0)<1 / \alpha$ and $h^{\prime \prime}(0)>81 /(4 \alpha)-3 h^{\prime}(0) / \alpha$. Moreover, under our assumptions, if we consider an initial state with a positive number of infectious, the corresponding solution will approach a limit cycle, that is, will oscillate. As we assume rather general conditions on $h$ at zero, our result applies to a wide class of nonlinearities.

Theorem 3. Assume that $h^{\prime \prime}(I)<0$ and $h^{\prime \prime \prime}(I)>0, \forall I$. If $h^{\prime}(0)<\frac{1}{\alpha}$ and $h^{\prime \prime}(0)>\frac{81}{4 \alpha}-\frac{3}{\alpha} h^{\prime}(0)$ then there exist $R_{1}$ and $R_{2}, 1<R_{1}<R_{2}$, such that

(a)

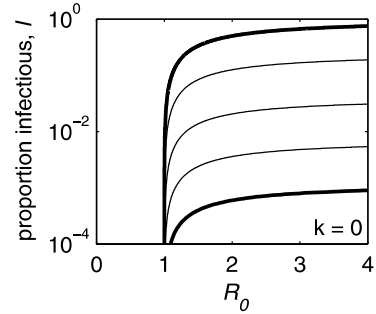

(b)

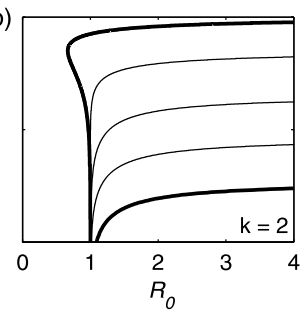

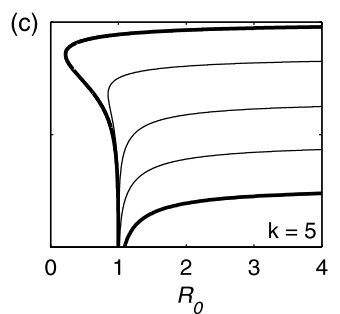

Fig. 3. Equilibrium branches for the (TI) system. The transmission coefficient is $r=$ $R_{0}+R_{1} k I /(1+k I)$ with $R_{1}=2$ and three values of $k$ : (a) $k=0$; (b) $k=2$; (c) $k=5$. The five curves within each plot correspond to different values of $\sigma$ : from the lowest to the highest curve, we have $\sigma=0,0.006,0.04,0.25,1$ 
for each $R_{0}$ satisfying $R_{1}<R_{0}<R_{2}$ system (TI) admits at least one limit cycle surrounding the nontrivial equilibrium. Moreover each solution with initial condition $\left(S_{0}, I_{0}\right)$ with $I_{0}>0$ and different from the nontrivial equilibrium has a limit cycle as $\omega$-limit and hence oscillates.

Proof. First of all, we can prove that for each $R_{0}>1$ the set $I=0$ is a uniform repeller from which follows that system $(T I)$ is uniformly persistent (for the definitions see [7] and [16]). To prove this fact we can use the method explained in [16] and based on the application of a theorem of Fonda [7] using as function $P(S, I)=I$.

Note that for each $R_{0}>1$ there exists a unique nontrivial equilibrium. Moreover the determinant $(\operatorname{det} J)$ and the trace $(\operatorname{tr} J)$ of the Jacobian matrix evaluated at the nontrivial equilibrium satisfy

$$
\operatorname{det} J>0 \Longleftrightarrow r^{\prime}(I)<\frac{\alpha}{(\alpha-I)^{2}}
$$

and

$$
\operatorname{tr} J>0 \Longleftrightarrow r^{\prime}(I)>\frac{\alpha^{3}}{I(\alpha-I)^{2}}
$$

Under our assumptions, there are $R_{1}$ and $R_{2}, 1<R_{1}<R_{2}$, such that for each $R_{0}$ satisfying $R_{1}<R_{0}<R_{2}$ the Jacobian matrix evaluated at the nontrivial equilibrium satisfies det $J>0$ and $\operatorname{tr} J>0$. Hence for each $R_{0}$ satisfying $R_{1}<R_{0}<R_{2}$ the nontrivial equilibrium is a repeller.

At this point the result is a consequence of the Poincaré-Bendixson theorem taking into account that the system is uniformly persistent.

In what concerns the stability of the nontrivial equilibria we have the following result where we set $\alpha=\alpha(\sigma)$,

Theorem 4. Assume that we are in the conditions of Theorem 3. If $h^{\prime \prime}(I)-\frac{\alpha^{3}(3 I-\alpha)}{I^{2}(\alpha-I)^{3}}$ admits at most one zero in the interval [0, $\alpha / 3]$, then there exist $I_{1}$ and $I_{2}$ such that the Jacobian matrix evaluated at the corresponding nontrivial equilibrium has two pure imaginary eigenvalues $\pm \beta_{j} i, j=1,2$. Moreover let

$$
\begin{aligned}
\sigma\left(I_{j}\right)= & r^{\prime \prime \prime}\left(I_{j}\right) \frac{I_{j}\left(\alpha-I_{j}\right)}{\alpha}+r^{\prime \prime}\left(I_{j}\right)\left(\frac{3\left(\alpha-I_{j}\right)}{\alpha}-2 \alpha-I_{j}\right)-6 \frac{\alpha^{4}}{I_{j}^{2}\left(\alpha-I_{j}\right)^{2}} \\
& +2 \frac{\alpha^{3}}{I_{j}^{2}\left(\alpha-I_{j}\right)}-\frac{\alpha}{\beta_{j}^{2}}\left(\frac{r^{\prime \prime}\left(I_{j}\right) I_{j}\left(\alpha-I_{j}\right)}{\alpha}-\frac{2 \alpha^{4}}{I_{j}\left(\alpha-I_{j}\right)^{2}}\right)^{2} \\
& +\left(\frac{\beta_{j}}{\alpha}-\frac{\alpha}{\beta_{j}}\right)\left(\frac{\alpha^{3}}{I_{j}\left(\alpha-I_{j}\right)}+\frac{\alpha}{I_{j}}\right)\left(\frac{r^{\prime \prime}\left(I_{j}\right) I_{j}\left(\alpha-I_{j}\right)}{\alpha}-\frac{2 \alpha^{4}}{I_{j}\left(\alpha-I_{j}\right)^{2}}\right)
\end{aligned}
$$

If $\sigma\left(I_{i}\right)<0, i=1,2$ then letting $R_{i}=R_{0}\left(I_{i}\right)$ we have

i) for each $1<R_{0}<R_{1}$ and for each $R_{0}>R_{2}$ the nontrivial equilibrium is locally asymptotically stable, 
ii) at $R_{0}=R_{1}$ there is a supercritical Hopf bifurcation and at $R_{0}=R_{2}$ there is a backward supercritical Hopf bifurcation, hence at $R_{0}=R_{1}$ a stable limit cycle appears and at $R_{0}=R_{2}$ a stable limit cycle disappears,

iii) for each $R_{1}<R_{0}<R_{2}$ the nontrivial equilibrium is unstable.

Remark. We note that, as a consequence of the previous results, the existence of backwards bifurcations or of oscillations is strongly related with the value of $h^{\prime}(0)$. Moreover, even if theoretically possible, it is unlikely that backwards bifurcations will be noticeable in the SIR model. In the case of the $S I S$ model, oscillations cannot appear and this agrees with the results previously obtained in [24].

Figure 4 shows the equilibrium surfaces for the $(T I)$ system. There are two principal observations. The first is a confirmation that for large regions of parameter space (towards the $S I S$ limit) there are backward bifurcations, and possibly two alternative stable equilibria: endemic, and disease free. An unstable equilibrium (coloured yellow) separates the two. The nonlinear force of infection is able to create equilibria where high prevalence of infection is self-sustaining. This effect is not seen where immunity is more protective against reinfection (towards the SI R limit) because the maintenance of a high endemicity relies on an adequate supply of susceptibles, leading to our second principal observation. Significant regions of parameter space permit sustained oscillations (coloured green). Essentially this is due to the additional time delay in recovery of the susceptible fraction of the population, which stabilises the otherwise damped oscillations (coloured blue). Note that red and green areas do not directly abut.

The conditions for bistable equilibria dominate at the SIS extreme but appear to be verified within a cusp-like region in the $\left(R_{0}, \sigma\right)$-parameter space. This is known as a cusp catastrophe [27]. In a similar manner, the conditions for sustained oscillations dominate at the $S I R$ extreme but also penetrate the $\left(R_{0}, \sigma\right)$-parameter space. The two areas (yellow and green) are expected to meet at the so-called Bodganov-Takens $(B T)$ points [3]. Figure 5 shows an amplification of the equilibrium surface in the area of greatest proximity between yellow and green regions. The Figure was obtained with the function $h$ is as in (2), with $R_{1}=2$ and two values

(a)

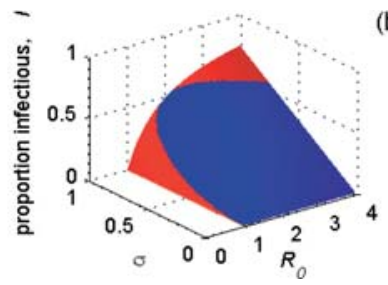

(b)

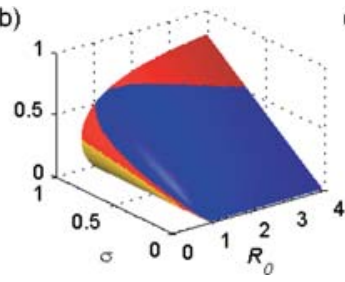

(c)

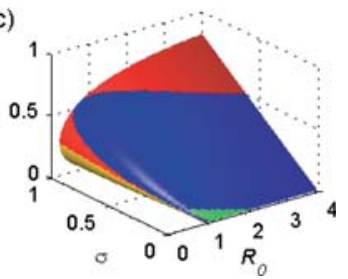

Fig. 4. Equilibrium surfaces for the (TI) system. The transmission coefficient is $r=R_{0}+$ $R_{1} k I /(1+k I)$ with $R_{1}=2$ and three values of $k$ : (a) $k=0$; (b) $k=2$; (c) $k=5$. Blue indicates that convergence takes the form of damped oscillations (the Jacobian has complex eigenvalues with negative real part), red indicates linear decay (the Jacobian has real negative eigenvalues), and yellow and green indicate that the equilibrium is unstable (the Jacobian has eigenvalues with positive real part - real eigenvalues in yellow and complex in green). Oscillatory dynamics are sustained around the green steady states 
(a)

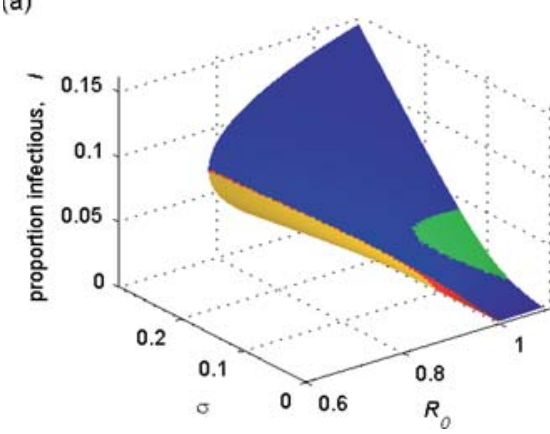

(b)

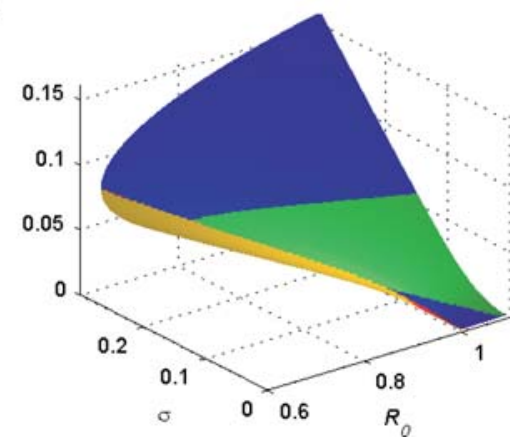

Fig. 5. Equilibrium surfaces for the $(T I)$ system showing the emergence of Bogdanov-Takens $(B T)$ points. The transmission coefficient is $r=R_{0}+R_{1} k I /(1+k I)$ with $R_{1}=2$ and two values of $k$ : (a) $k=5$ as in figure 4(c) showing no $B T$ points; (b) $k=7$ revealing two $B T$ points

of $k: k=5$ as in Figure 4(a) showing no $B T$ points; and $k=7$ as in Figure 4(b) revealing two $B T$ points (where the blue, green and yellow regions meet). In the next theorem we give a condition for the existence of $B T$ points.

Theorem 5. Assume that there is $\alpha^{*}=\alpha^{*}\left(\sigma^{*}\right)$ such that $r^{\prime}\left(\alpha^{* 2}\right)=\frac{1}{\alpha^{*}\left(1-\alpha^{*}\right)^{2}}$.

Then $(S, I)=\left(1-\alpha^{*}, \alpha^{* 2}\right)$ is a point of Bogdanov-Takens if

$$
r^{\prime \prime}\left(\alpha^{* 2}\right) \neq \frac{3 \alpha^{*}-1}{\alpha^{* 3}\left(1-\alpha^{*}\right)^{3}} \text { and } r^{\prime \prime}\left(\alpha^{* 2}\right) \neq \frac{\left(3 \alpha^{*}-1\right)\left(\alpha^{* 2}-6 \alpha^{*}+3\right)}{\alpha^{* 3}\left(\alpha^{* 2}+3 \alpha^{*}-2\right)\left(\alpha^{*}-1\right)^{3}} \text {. }
$$

Proof. Under the conditions of the theorem we can apply the result in [3, Proposition 4.3.2].

Remark. Note that if (7) is not satisfied for $r$, it is satisfied for a perturbation of $r$, $r_{\epsilon}(I)=r(I)+\frac{\epsilon}{2} I\left(I-\alpha^{* 2}\right)^{2}$, with $\epsilon$ sufficiently small.

If we choose $h$ as in (2) with $R_{1}=2$ and $k=7$, for example, we find two values of $\alpha$ for which Theorem 5 applies. Figure 6 is a schematic representation of the dynamical behaviour of system $(T I)$ in vicinity of the $B T$ points (this can be viewed as a projection of Figure $5(\mathrm{~b})$ onto the $\left(R_{0}, \sigma\right)$-plane). Regions $\mathrm{A}, \mathrm{B}, \mathrm{I}, \mathrm{J}$, are characterised by stable endemic steady states and correspond to blue or red areas of the surface in Figure 5(b). Regions C, D, E, F, G, H, are characterised by epidemic behaviour and constitute the green area of Figure 5(b). On entering the green area, the endemic steady states loose stability to a periodic orbit (Hopf bifurcation) and further in, the periodic orbit is destroyed by collapsing with a saddle point (homoclinic bifurcation). This generates a tendency for intermittent behaviour in regions E, F: the system spends long periods of time near the disease-free equilibrium but, unless the infection is stochastically extinct, there is a strong potential for recurrent 


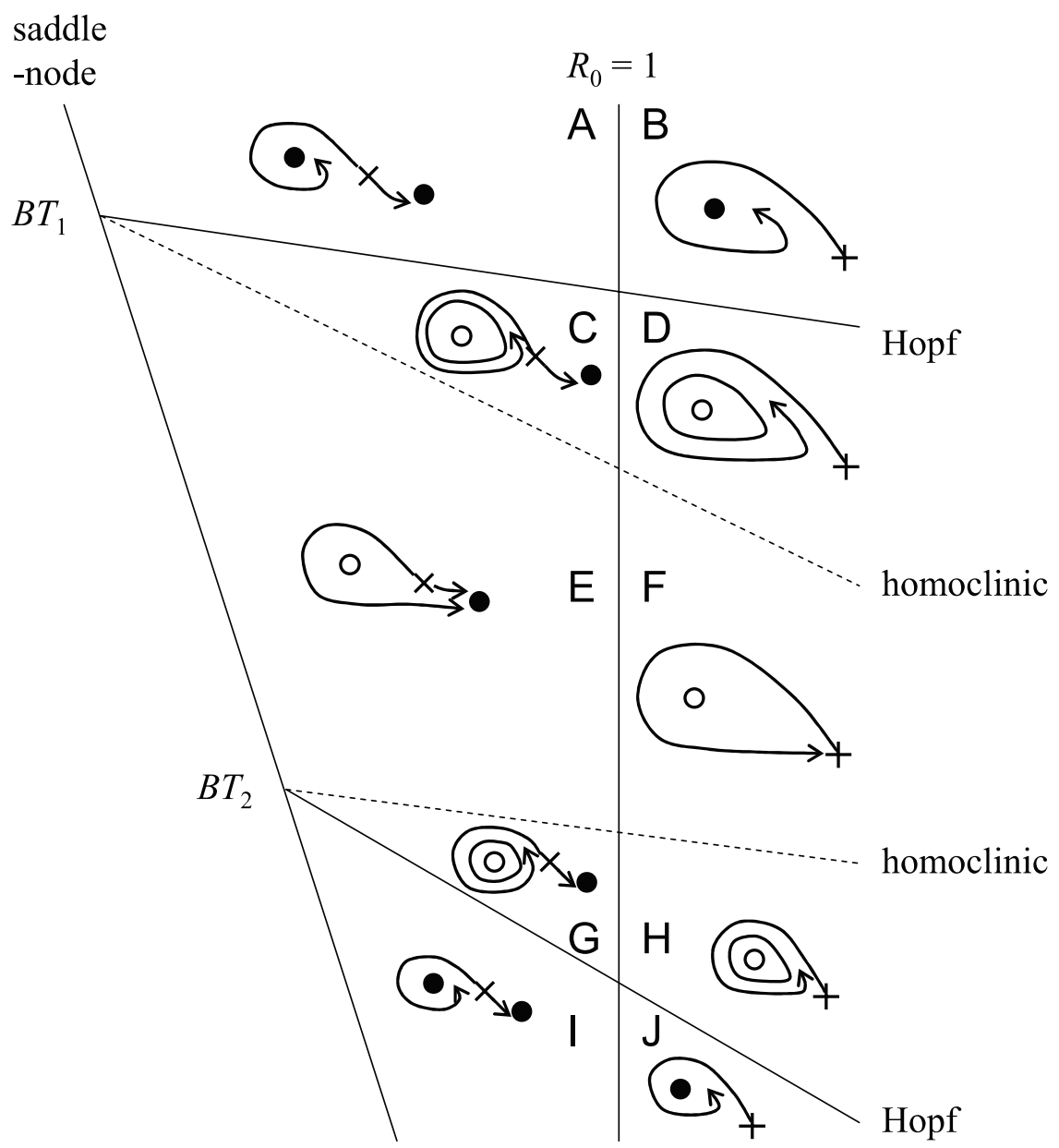

Fig. 6. Schematic representation of the behaviour associated with the two BogdanovTakens points $\left(B T_{1}\right.$ and $\left.B T_{2}\right)$ detected in figure 5(b). The diagram illustrates the partition of the $\left(R_{0}, \sigma\right)$ space into regions of qualitatively distinct dynamics. In each region there is a phase portrait representing the essential features of the dynamics. Full and open dots represent stable and unstable steady states respectively, crosses represent saddle points, closed loops represent periodic orbits, and lines with arrows represent the flow. There is a steady state on the right-bottom corner of each phase portrait corresponding to the disease-free equilibrium $(S, I)=(1,0)$. Note that this is stable for $R_{0}<1$ and a saddle for $R_{0}>1$. The homoclinic bifurcations are known to emanate from the $B T$ points as indicated, but their exact path has not been followed by our analysis. For this reason they are marked as dashed lines. Regions C, D, G, H are characterised by sustained periodic epidemics. As we move towards regions $\mathrm{E}, \mathrm{F}$, the interepidemic periods increase until the infection is apparently extinct. However, unless stochastic extinction occurs, recurrences are possible at any time

epidemics. These are expected to occur spontaneously when $R_{0}>1$, and under small peturbations when $R_{0}<1$. Intermittent behaviour associated with homoclinic cycles can have dramatic effects on the long term behaviour of epidemiological 
systems, and should be investigated in the context of emergent and re-emergent diseases. Here this behaviour is linked to two $B T$ points whose detection requires a continuum of models between the $S I R$ and $S I S$ extremes. The $B T$ points are the organizing centres for the dynamics, but the practical implications spread over large regions of parameter space.

\section{The partial immunity model}

In the case of system $(P I)$, the branch of nontrivial equilibria is not described analytically in the general case due to the complexity of the function $\eta_{P}$. Using the nonlinear function $h$ defined in (2) we have calculated the steady states numerically as well as their stability. The results are shown in Figures 7 and 8 for $R_{1}=2$ and three values of $k=0,2$ and 5 . As in system ( $T I)$, backward bifurcations occur and this becomes more evident as $k$ increases. Recall that a backward bifurcation implies bistability of an endemic equilibrium and the disease-free equilibrium for a range of $R_{0}<1$. In addition, now we can have bistability of two endemic equilibria (high and low) and this is evident in Figures 7(c) and 8(c). Also as in system $(T I)$, we have the possibility of sustained oscillations as marked by the green area

(a)

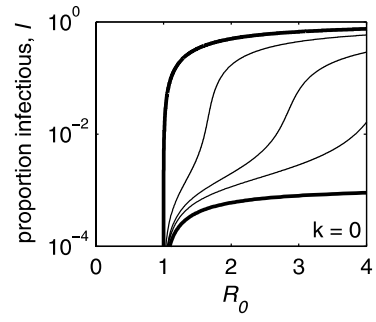

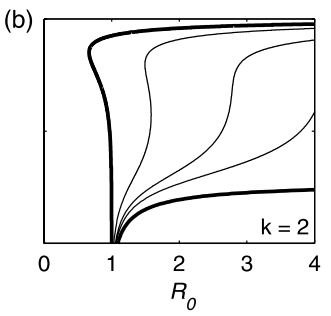

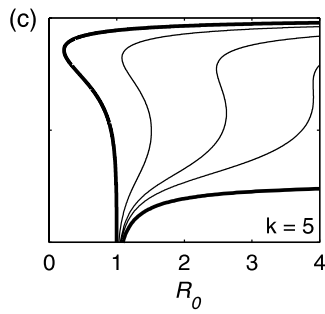

Fig. 7. Equilibrium branches for the $P I$ system. The transmission coefficient is $r=R_{0}+$ $R_{1} k I /(1+k I)$ with $R_{1}=2$ and three values of $k$ : (a) $k=0$; (b) $k=2$; (c) $k=5$. The five curves within each plot correspond to different values of $\sigma$ : from the lowest to the highest curve, we have $\sigma=0,0.24,0.35,0.6,1$

(a)

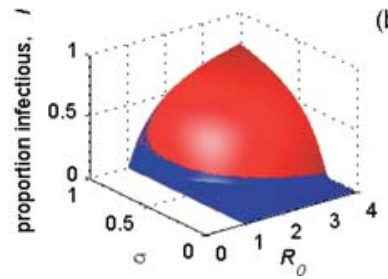

(b)

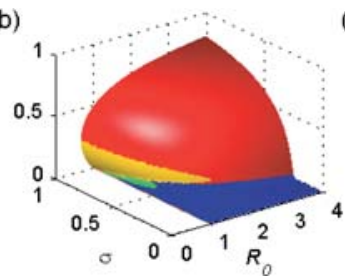

(c)

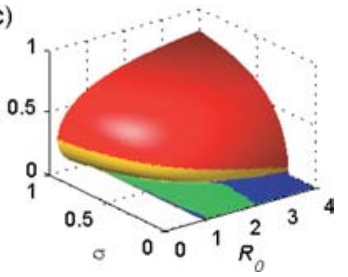

Fig. 8. Equilibrium surfaces for the $P I$ system. The transmission coefficient is $r=R_{0}+$ $R_{1} k I /(1+k I)$ with $R_{1}=2$ and three values of $k$ : (a) $k=0$; (b) $k=2$; (c) $k=5$. Blue indicates that convergence takes the form of damped oscillations (the Jacobian has complex eigenvalues with negative real part), red indicates linear decay (the Jacobian has real negative eigenvalues), and yellow and green indicate that the equilibrium is unstable (the Jacobian has eigenvalues with positive real part - real eigenvalues in yellow and complex in green). Oscillatory dynamics are sustained around the green steady states 
in Figure 8(b,c). Note the sharp rise in incidence across the reinfection threshold ([10]).

\section{The SIR and SIS models with nonlinear incidence}

In this section we analyse further the models previously introduced in the limit cases when $\sigma=0$ or $\sigma=1$ assuming $h^{\prime \prime}(I)<0, \forall 0 \leq I \leq 1$. These models are the SIR and SIS models with nonlinear incidence. In the case $\sigma=1$ we can identify the classes $S$ and $R$ and consider a one-dimensional model as we have $S+I=1$. The corresponding equations are

$$
\begin{aligned}
& (S I R)\left\{\begin{array}{l}
\dot{S}=e-\Lambda S-e S \\
\dot{I}=\Lambda S-I
\end{array}\right. \\
& (S I S) \quad \dot{I}=\Lambda(1-I)-I .
\end{aligned}
$$

As previously demonstrated, the disease-free state $(S=1, I=0)$ is an equilibrium for both systems for all values of $R_{0}$. Moreover this equilibrium is locally asymptotically stable for $R_{0}<1$ and unstable for $R_{0}>1$. For endemic steady states we have $S=\frac{1}{r(I)}$ and $I$ is a solution of an equation that is model dependent

$$
\begin{aligned}
& (S I R \text { model }): r(I)=\frac{e}{e-I} \\
& (S I S \text { model }): r(I)=\frac{1}{1-I} .
\end{aligned}
$$

In what concerns the $S I R$ model, we can rewrite Theorems 3 and 4 replacing $\alpha$ with $e$. For example, from Theorem 3 follows

Theorem 6. Consider the SIR model and assume that $h^{\prime \prime \prime}(I)>0, \forall I . I f h^{\prime}(0)<\frac{1}{e}$ and $h^{\prime \prime}(0)>\frac{81}{4 e}-\frac{3}{e} h^{\prime}(0)$ then there exist $R_{1}$ and $R_{2}, 1<R_{1}<R_{2}$, such that for each $R_{0}$ satisfying $R_{1}<R_{0}<R_{2}$ the system admits at least one limit cycle surrounding the nontrivial equilibrium. Moreover each solution with initial condition $\left(S_{0}, I_{0}\right)$ with $I_{0}>0$ and different from the nontrivial equilibrium has a limit cycle as $\omega$-limit and hence oscillates.

With respect to the SIS model, and using the notations of Theorem 2, we have

Theorem 7. Consider the SIS model. If $h^{\prime}(0)>1$ then there exists $R_{1}<1$ such that for each $R_{0}$ satisfying $R_{1}<R_{0}<1$ the nontrivial equilibrium corresponding to low prevalence of infection is unstable while for each $R_{0}>R_{1}$ the one corresponding to high prevalence is stable. If $h^{\prime}(0)<1$ then the nontrivial equilibrium is stable. 
Proof. Note that $g(I):=h^{\prime}(I)-\frac{1}{(1-I)^{2}}$ is a decreasing function of $I$.

First of all assume $h^{\prime}(0)>1$. Then $g(0)>0$ and if $I_{1}$ is such that $R_{0}\left(I_{1}\right)=R_{1}$, $g\left(I_{1}\right)=0$. Hence the determinant of the Jacobian matrix $J$ evaluated at the low prevalence nontrivial equilibrium is negative while it is positive at the one corresponding to high prevalence. Also at the high prevalence equilibrium $r^{\prime}(I)<$ $\frac{1}{(1-I)^{2}}<\frac{1}{I(1-I)^{2}}$ and hence $\operatorname{tr} J<0$.

If $h^{\prime}(0)<1$, for all $I>0$ we have $h^{\prime}(I)<\frac{1}{(1-I)^{2}}<\frac{1}{I(1-I)^{2}}$. Thus the determinant of the Jacobian matrix $J$ evaluated at the nontrivial equilibrium is positive while the corresponding trace is negative. The nontrivial equilibrium is in this case stable.

\section{Discussion}

Mathematical models with nonlinear force of infection have been studied by many authors $([14,22,12,24,1])$. In several of these papers (see for example [14,22]) simple models with a particular nonlinear force of infection are proposed and analysed. In [12], a $S E I R S$ (susceptible-exposed-infectious-removed-susceptible) with a general nonlinear incidence rate is analysed - a theorem about the existence of multiple equilibria is given in the general case but the stability and the existence of oscillations are obtained only in the case of the incidence $R_{0} I^{p} /\left(1+\alpha I^{q}\right)$.

A $S I V$ (susceptible-infectious-perfectly vaccinated) model with nonlinear incidence and nonconstant population was analysed in [1]. The authors analyse the model and give some examples in which backwards bifurcations or oscillations appear. In [24] a SI S model with a general nonlinear incidence function is proposed and it is proved the existence of multiple stable equilibria, backwards bifurcations and hysteresis, but in this case oscillations do not exist. The model consists on a Volterra integral equation which includes ordinary and delay differential equations as special cases.

Here we increment the transmission coefficient, $R_{0}$, with a strictly increasing function $h(I)$. Immunity spans the whole range of models from $S I R$ to $S I S$ by means of one parameter families representing either temporary immunity $(T I)$ or partial immunity $(P I)$. The results that we obtain should apply in a large number of situations. In particular, the existence of backwards bifurcations obtained in Theorem 1 or the sustainability of oscillations in Theorem 3 are common in models with nonlinear incidence. Hence it is natural to expect catastrophic phenomena and sustained oscillations without external forcing (such as seasonality, for example). Analytical results are illustrated and extended by numerical solutions. For this purpose we specify a functional form for $h$ which is controlled by two parameters: $R_{1}$ sets the magnitude of variation in the transmission coefficient (between $R_{0}$ and $\left.R_{0}+R_{1}\right)$; and $k$ sets the steepness at $I=0\left(\right.$ as $\left.h^{\prime}(0)=R_{1} k\right)$. By numerical inspection we confirm that backward bifurcations are dominant at the $S I S$ limit while sustained oscillations strive at the $S I R$ limit. Furthermore, we find Bogdanov-Takens points and homoclinic bifurcations in both $(T I)$ and $(P I)$ model families. 
The issue of averaging characteristics of transmission to incorporate the force of infection is very subtle. Models based on standard mass-action transmission assume that the ratio (force of infection / prevalence of infection) is insensitive to changes in prevalence levels. However, if the probability of infection increases with the intensity of exposure, then this ratio should increase with the prevalence of infectious individuals. A wealth of mathematical models shows that the shape of this relationship is an important determinant of qualitative and quantitative epidemiological properties, such as whether the system converges to stationary or oscillatory equilibria and the level of such equilibria. Although the importance of this relationship has been theoretically recognised for at least 20 years [14,5], applications to real disease problems are rare and, probably necessarily, disease specific $[11,17]$.

The estimation of a nonlinear relationship between prevalence of infection and the transmission capacity (or coefficient) from epidemiological data is difficult. This requires some form of data stratification [6] and involves a complex of factors. For some infections data suggests that severity of disease increases with intimacy of contact or, more generally, with the intensity of exposure to the infectious agent (e.g. measles [26], polio [20], foot-and-mouth disease [8,2]). But dose of exposure may also be important for subsequent infectiousness as suggested by experiments on bovine tuberculosis $[18,19]$. Intensity and frequency of exposure is also likely to affect the potency and duration of protective immunity.

There is also a circularity problem in terms of estimating model parameters [17]. If linearity in the force of infection is assumed, then, generally, in these frameworks $R_{0}=1 / S$ (the reciprocal of the proportion susceptible). Consequently, most current estimates of $R_{0}$ are outside the regions of complicated dynamics (i.e. $>3$ ). One consequence is that models including non-linear incidence are assumed to be of mathematical, but not practical, interest. But, the relationships between the basic reproduction number and prevalence of infection (Figs 3 and 7) are highly dependent on the other parameters. The estimation of $R_{0}$ is not model independent, so that many current estimates of $R_{0}$ may be incorrectly over-estimated (since they implicitly assume $k=0$ ).

We consider that the risk of infection increases with the intensity of exposure, and that infection induces some protective immunity that reduces the risk of reinfection (by providing full protection temporarily, or partial protection permanently). Extensions to this work should address more deeply the relationships between patterns of infection and the resulting immunity, which would then affect the patterns of reinfection, and so on. This issue is pertinent for several childhood diseases that are being considerably reduced by vaccination programmes but persist at reduced incidence.

\section{References}

1. Alexander, M.E., Moghadas, S.M.: Periodicity in an epidemic model with a generalized non-linear incidence. Math. Biosciences, 189 , 75-96 (2004)

2. Alexandersen, S., Quan, M., Murphy, C., Knight, J., Zhang, Z.: Studies of quantitative parameters of virus excretion and transmission in pigs and cattle experimentally infected with foot-and-mouth disease virus. J. Comp. Path., 129 , 268-282 (2003) 
3. Arrowsmith, D.K., Place, C.M.: An introduction to Dynamical Systems. Cambridge: Cambridge University Press, 1994

4. Diekmann, O., Heesterbeek, J.A.P., Metz, J.A.J.: On the definition and the computation of the reproduction number R0 in models for infectious diseases in heterogeneous populations. J. Math. Biology 28, 365-382 (1991)

5. Dushoff, J.: Incorporating Immunological Ideas in Epidemiological Models. J. Theor. Biol. 180, 181-187 (1996)

6. Farrington, C.P., Kanaan, M.N., Gay, N.J.: Estimation of the basic reproduction number of infectious diseases from age-stratified serological survey data. Applied Statistics 50, 251-292 (1996)

7. Fonda, A.: Uniformly persistent semidynamical systems. Proc. Amer. Math. Soc. 104, $111-116(1988)$

8. French, N.P., Kelly, L., Jones, R., Clancy, D.: Dose-response relationships for foot and mouth disease in cattle and sheep. Epidemiol. Infect. 128 , 325-332 (2002)

9. Gomes, M.G.M., White, L.J., Medley, G.F.: Infection, reinfection and vaccination under sub-optimal immune protection: epidemiological perspectives. J. Theor. Biol. 228, 539549 (2004)

10. Gomes, M.G.M., White, L.J., Medley, G.F.: The Reinfection Threshold. J. Theor. Biol. (in press)

11. Greenhalgh, D., Diekmann, O., de Jong, M.C.M.: Subcritical endemic steady states in mathematical models for animal infections with incomplete immunity. Math. Biosci. $\mathbf{1 6 5}, 1-25$ (2000)

12. Hethcote, H.W., van den Driessche, P.: Some epidemiological models with nonlinear incidence. J. Math. Biology, 29, 271-287 (1991)

13. Kermack, W.O., McKendrick, A.G.: A contribution to the mathematical theory of epidemics. Proc. R. Soc. Lond. A 115 , 700-721 (1927)

14. Liu, W., Hethcote, H.W., Levin, S.A.: Dynamical behavior of epidemiological models with nonlinear incidence rates. J. Math. Biology, 25, 359-380 (1987)

15. McCallum, H., Barlow, N., Hone, J.: How should pathogen transmission be modelled?. Trends Ecol. Evol. 16, 295-300 (2001)

16. Margheri, A., Rebelo, C.: Some examples of persistence in epidemiological models. J. Math. Biology, 46, 564-570 (2003)

17. Medley, G.F., Lindop, N.A., Edmunds, W.J., Nokes, D.J.: Hepatitis-B virus endemicity: heterogeneity, catastrophic dynamics and control. Nature Medicine 7, 619-624 (2001)

18. Morrison, W.I., Bourne, F.J., Cox, D.R., Donnelly, C.A., Gettinby, G., McInerney, J.P., Woodroffe, R.: Pathogenesis and diagnosis of infections with Mycobacterium bovis in cattle. Independent Scientific Group on Cattle TB. The Veterinary Record 146, 236-242 (2000)

19. Neill, S.D., Hanna, J., O’Brien, J.J., McCracken, R.M.: Excretion of Mycobacterium bovis by experimentally infected cattle. The Veterinary Record 123, 340-343 (1988)

20. Nielsen, N.M., Aaby, P., Wohlfahrt, J., Pedersen, J.B., Melbye, M., Molbak, K.: Intensive exposures as a risk factor for severe polio: a study of multiple family cases. Scand. J. Infect. Dis. 33, 303-305 (2001)

21. Regoes, R.R., Ebert, D., Bonhoeffer, S.: Dose-dependent infection rates of parasites preduce the Allee effect in epidemiology. Proc. R. Soc. Lond. B 269, 271-279 (2002)

22. Ruan, S., Wang, W.: Dynamical behavior of an epidemic model with a nonlinear incidence rate. J. Differential Equations, 188, 135-163 (2003)

23. Tuckwell, H.C., Toubiana, L., Vibert, J.-F.: Spatial epidemic network models with viral dynamics. Phys. Rev. E 57, 2163-2169 (1998)

24. van den Driessche, P., Watmough, J.: A simple SIS epidemic model with a backward bifurcation. J. Math. Biology, 40, 525-540 (2000) 
25. White, L.J., Medley, G.F.: Microparasite population dynamics and continuous immunity. Proc. R. Soc. Lond., B 265, 1977-1983 (1998)

26. Whittle, H.C., Aaby, P., Samb, B., Jensen, H., Bennett, J., Simondon, F.: Effect of subclinical infection on maintaining immunity against measles in vaccinated children in West Africa. The Lancet 353, 98-102 (1999)

27. Zeeman, E.C.: Catastrophe Theory. Selected Papers 1972 - 1977. Addison-Wesley Publ. Co., 1977 